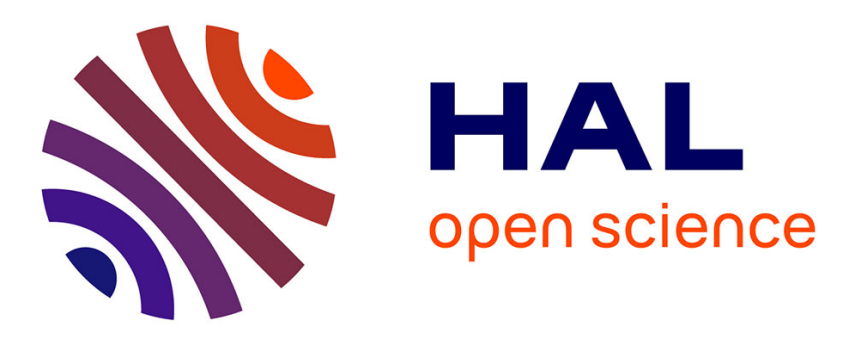

\title{
Bubble dynamics inside an outgassing hydrogel confined in a Hele-Shaw cell
}

Florence Haudin, Xavier Noblin, Yann Bouret, Médéric Argentina, Christophe Raufaste

\section{- To cite this version:}

Florence Haudin, Xavier Noblin, Yann Bouret, Médéric Argentina, Christophe Raufaste. Bubble dynamics inside an outgassing hydrogel confined in a Hele-Shaw cell. Physical Review E , 2016, 94, pp.023109. 10.1103/PhysRevE.94.023109 . hal-01358063

\section{HAL Id: hal-01358063 https://hal.science/hal-01358063}

Submitted on 30 Aug 2016

HAL is a multi-disciplinary open access archive for the deposit and dissemination of scientific research documents, whether they are published or not. The documents may come from teaching and research institutions in France or abroad, or from public or private research centers.
L'archive ouverte pluridisciplinaire HAL, est destinée au dépôt et à la diffusion de documents scientifiques de niveau recherche, publiés ou non, émanant des établissements d'enseignement et de recherche français ou étrangers, des laboratoires publics ou privés. 


\title{
Bubble dynamics inside an outgassing hydrogel confined in a Hele-Shaw cell
}

\author{
Florence Haudin, ${ }^{1,2,3}$ Xavier Noblin, ${ }^{1}$ Yann Bouret, ${ }^{1}$ Médéric Argentina, ${ }^{4,5}$ and Christophe Raufaste ${ }^{1, *}$ \\ ${ }^{1}$ Université Côte d'Azur, CNRS, LPMC, France \\ ${ }^{2}$ Institut Langevin Laboratoire Ondes et Images, LOA, \\ UMR CNRS 7587-ESPCI, 5 rue Jussieu, 75005 Paris, France \\ ${ }^{3}$ Laboratoire de Physique et Mécanique des Milieux Hétérogènes (PMMH), \\ UMR CNRS 7636-ESPCI-UMPC-UPD, 10 rue Vauquelin, 75005 Paris, France \\ ${ }^{4}$ Université Côte d'Azur, CNRS, INLN, France \\ ${ }^{5}$ Institut Universitaire de France, 75005 Paris, France
}

\begin{abstract}
We report an experimental study of bubble dynamics in a non-Newtonian fluid subjected to a pressure decrease. The fluid is a hydrogel, composed of water and a synthetic clay, prepared and sandwiched between two glass plates in a Hele-Shaw geometry. The rheological properties of the material can be tuned by the clay concentration. As the imposed pressure decreases, the gas initially dissolved in the hydrogel triggers bubble formation. Different stages of the process are observed : bubble nucleation, growth, interaction and creation of domains by bubbles contact or coalescence. Initially bubble behave independently. They are trapped and advected by the mean deformation of the hydrogel, and the bubble growth is mainly driven by the diffusion of the dissolved gas through the hydrogel and its outgassing at the reactive-advected hydrogel-bubble interface. In this regime, the rheology of the fluid does not play a significant role on the bubble growth. A model is proposed and gives a simple scaling that relates the bubble growth rate and the imposed pressure. Carbon dioxide is shown to be the gas at play and the hydrogel is degassing at the millimeter scale as a water solution does at a smaller scale. Later on, bubbles are not independent anymore. The growth rate decreases and the morphology becomes more anisotropic as bubbles interact because they are separated by a distance smaller than the individual stress field extension. Our measurements show that the interaction distance scales as the bubbles size.
\end{abstract}

PACS numbers: 47.55.D- 87.19.rh 82.40.Ck

\section{INTRODUCTION}

Bubble growth inside a non-Newtonian fluid mixes numerous fundamental and applied fields. Among them, failure in amorphous solids results from cavitation inside a viscoplastic zone at the end of a crack tip [1]. Cavitation and bubble growth occur inside adhesives under tension $[2,3]$, or inside a drying gel [4]. Growing a single bubble at the tip of syringe is used as a local probe to characterize the mechanical properties of soft materials and biological tissues [5-7]. Bubble nucleation and growth can also be provoked by pulsed lasers for cutting biological tissues [8].

The case of an oversaturated fluid containing dissolved gases leads to diffusion-fed bubble growth mechanisms. After fermentation of wheat flour dough $[9,10]$ or the processing of polymer foams [11], the morphologies of the bubbles and of their formed network have a direct impact on the properties of the final product. In geology, it is encountered in the outgassing of volcanic magmas [12] and mantle melts [13].

In newtonian fluids, the bubble growth from dissolved gases also happens in multiple situations, and in particular in carbonated beverages. When a champagne bottle is opened, the initially pressurized wine undergoes a drastic pressure decrease : bubble growth is then observed (see

\footnotetext{
* corresponding author : Christophe.Raufaste@unice.fr
}

a review on the topic [14]). The rheological fluid properties are then close to water and the spatial distribution of bubbles, which is directly correlated to nucleation sites, is rapidly influenced by gravity and finally leads to specific bubble columns. Theoretically, Epstein and Plesset [15] studied the diffusion-fed growth of a spherical bubble in an oversaturated liquid. Accounting for the liquid-bubble interface advection and the effect of surface tension, they calculated the radial concentration of the dissolved species around the bubble by a competition between the dissolved species diffusion and their outgassing at the liquid-gas interface. Several studies extended the case of Newtonian liquids [16] to more complex rheological behaviors [17-20].

The models of a single bubble growth deal with the spherical geometry which seems in agreement with ductile-like materials [19]. This is the case as long as bubbles does not interact. At most, bubbles enter into contact or percolate and can lead to very complex and anisotropic structures $[2,10]$. It can be noted that the geometry can evolve from the regular sphere to a fracture-like anisotropic shape inside brittle materials when the bubble growth time scale is faster than the stress relaxation time $[6,21]$.

In this context, we report an experimental study of the diffusion-fed bubble growth inside a hydrogel. Such a gel exhibits both a non-Newtonian behavior and contains a significant amount of dissolved gases at standard pressure-temperature conditions. The supersatura- 
tion is triggered by a pressure decrease and the hydrogel confinement between two glass plates in a Hele-Shaw geometry enables a direct imaging of the bubbles growth and morphology in a quasi 2D geometry. The originality of this work is related to the concomitant focus on both the growth rate and morphology of the bubbles, and to the study of the onset of the bubble interaction.

As the pressure decreases, bubbles nucleate and start to grow. The growth dynamics is twofold. In a first regime, the bubbles behave independently and the growth rate is only related to the imposed pressure regardless of the bubble size and history. The growth rate is driven by the gas concentration profile in the vicinity of the bubble. It depends on the diffusive properties in the hydrogel bulk and the saturation equilibrium at the hydrogel-bubble interface. This regime is denoted "diluted" since bubbles are too far away from each other to interact. Bubbles morphology is homogeneous and rather isotropic regardless of the rheological properties of the hydrogel in our parameters range. In a second regime, denoted "dense", bubbles interact. This corresponds to gas fractions around $5-10 \%$. The growth rate decreases due to a competition for the dissolved gases and the bubble morphology becomes more and more anisotropic with a significant effect of the hydrogel rheological properties. Bubbles of the same size begin to interact when the center-to-center bubble distance becomes comparable to the bubble size.

The article is organized as follows : Section II is dedicated to the experimental setup. Section III deals with the qualitative observations that are quantitatively described in section IV for the diluted regime and in section VI for the dense regime. A model for the diluted regime is presented in section $\mathrm{V}$.

\section{MATERIALS AND METHODS}

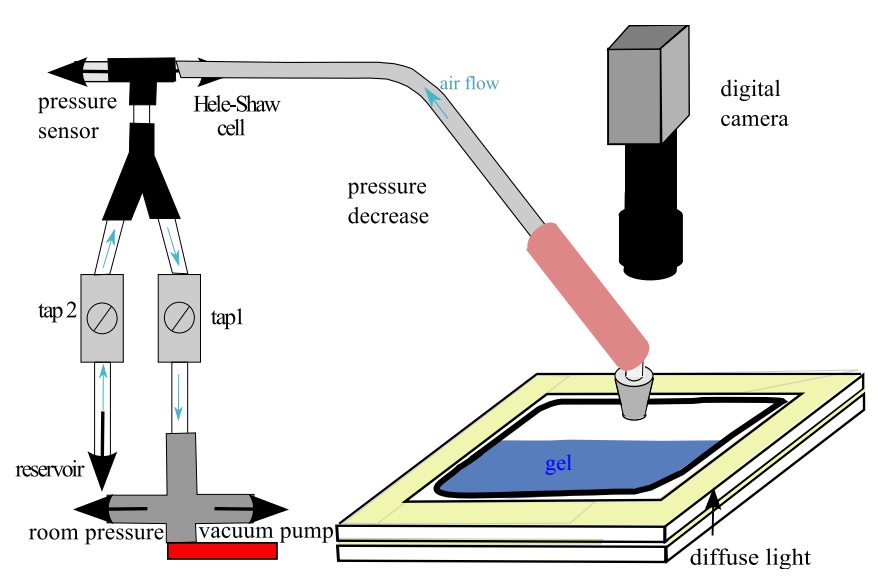

FiguRE 1. Representation of the experimental setup.

The setup consists in a Hele-Shaw cell filled with a hydrogel and connected to a vacuum pump to drive the pressure decrease (Fig. 1). The cell is built with two glass

\begin{tabular}{|c|c|c|c|}
\hline $\begin{array}{c}\text { hydrogel } \\
\text { composition }\end{array}$ & $\begin{array}{c}\text { elastic modulus } E \\
(\mathrm{~Pa})\end{array}$ & $\begin{array}{c}\text { yield stress } \sigma_{Y} \\
(\mathrm{~Pa})\end{array}$ & $\begin{array}{c}\text { viscosity } \eta \\
(\mathrm{Pa} . \mathrm{s})\end{array}$ \\
\hline \hline $3 \%$ & $2 \pm 2$ & $0.2 \pm 0.2$ & $0.025 \pm 0.005$ \\
\hline $4 \%$ & $500 \pm 50$ & $50 \pm 15$ & $0.012 \pm 0.002$ \\
\hline $5 \%$ & $1090 \pm 40$ & $115 \pm 15$ & $0.010 \pm 0.003$ \\
\hline
\end{tabular}

TABLE I. Rheological properties of the hydrogels measured by a cone plate rheometer (angle $1 \mathrm{deg}$ ). The Young modulus $E$ is determined by oscillatory shear measurements at $5 \mathrm{~Hz}$. The yield stress $\sigma_{Y}$ and the viscosity $\eta$ are obtained by fitting the stress-shear rate curve of continuous shear experiments by a Bingham fluid model.

plates $(200 \times 200 \times 10 \mathrm{~mm})$ clamped on both sides of a $1 \mathrm{~mm}$ thick waterproof spacer (in a sake of simplicity, the clamping system is not shown in Fig. 1). The thickness of the spacer was varied between 0.5 and $2 \mathrm{~mm}$, and this spacing did not affect qualitatively the bubble dynamics. In what follows, $1 \mathrm{~mm}$ is chosen as a good compromise to get a good contrast and prevent superposition of bubbles in the confining dimension. A hole is drilled through the upper plate to enable the cell filling and the vacuum pump connection.

Our hydrogels are made of distilled water and Laponite ${ }^{\circledR}$ RD, a synthetic clay manufactured by Rockwood. Composition of the hydrogel ranges between 0 and $5 \%$ of Laponite in mass. Laponite powder is added in 20 minutes to water under strong stirring to ensure a homogeneous dispersion and prevent flocculation. After 20 minutes of extra-stirring the mixture is still relatively liquid and is poured inside the cell. The higher the Laponite content, the higher the initial gelation of the hydrogel and small bubbles are trapped for the $5 \%$ hydrogel. For all compositions, $\mathrm{pH}$ measurements give a value around 10, which is coherent with the fact that the Laponite suspension behaves as a buffer solution. The cell is then kept at rest for 24 hours to ensure the complete gelation of the mixture. Concentrations smaller than $2 \%$ did not exhibit any gelling behavior. For higher concentrations, the hydrogel sustains its shape and does not flow under gravity. The visco-elasto-plastic properties are measured by a cone plate rheometer (Tab. I). The rigidity of the hydrogel changes significantly : both the yield stress and the elastic modulus vary over a factor 500 between the $3 \%$ and $5 \%$ compositions.

The pressure decrease is driven by a vacuum pump. It is connected to the cell through a tubing connection system made of a three-way valve and two taps (Fig. 1). The valve enables to start and stop the pressure decrease process. The first tap connects the cell and the pump while the second tap connects the cell with a closed and large reservoir initially at room pressure. The second tap generates a leak and decreases the efficiency of the vacuum pump. By configuring both taps, both the pressure decrease rate and the final pressure value are prescribed. The latter is always chosen larger than 30 mbar to prevent the water vaporization. A pressure sensor and 
an $\mathrm{I} / \mathrm{O}$ card record continuously the pressure evolution in the system during the experiment. This pressure corresponds to those imposed at the free interface of the hydrogel. Note that the cell is never completely filled (Fig. 1) so that the hydrogel does not enter the tubing connection system when it expands due to bubble growth.

Finally, the cell is placed on a transmitted light illumination system and the dynamics inside the cell is recorded with a digital camera (Fig. 1). Each bubble is characterized by its projected area $A$ based on the outermost contour. The growth rate is given by $d A / d t$.

\section{QUALITATIVE OBSERVATIONS}

The main features of the hydrogel evolution are represented in Fig. 2 for a $4 \%$ hydrogel. These concern bubble nucleation, growth, interaction and finally percolation of gaseous domains. The vacuum pump reduces the pressure from 1000 mbar to 50 mbar in typically 10 minutes. Once bubbles appear, they drift toward the pumping direction to account for the gas generation and the whole expansion of the hydrogel. Bubbles do not move inside the hydrogel matrix itself when gelation is strong enough. This occurs for composition larger than $3 \%$. We recorded a temperature decrease, at most $10^{\circ} \mathrm{C}$, inside the hydrogel during an experiment.

Around 500 mbar, nucleations start (Fig. 2a) and bubbles appear continuously leading to typical densities around a few bubbles $/ \mathrm{cm}^{2}$. While they are significantly smaller than the millimeter scale, it is difficult to observe a deviation from circularity. When their sizes approach the millimeter scale (Fig. 2bc), they develop anisotropy. Fewer supplementary nucleation events are observed when the system enters the dense regime (Sec. $\mathrm{VI}$ ).

When bubbles are far from each other, typically for bubbles separated from their neighbors within a few bubble radii (Sec. VI), they tend to elongate like ellipses in the direction perpendicular to the pumping direction. Their shape and growth rate are not influenced by their neighbors. This regime is called "diluted". Note that some isolated bubbles can be slightly oriented toward the pumping direction. In this regime, the smaller the pressure, the larger the growth rate of the bubbles. The higher the amount of the gelling agent, the more anisotropic the bubbles (Fig. 3).

When bubbles are close to each other (Fig. 2cd), they tend to grow in the direction of their neighbors. This characterizes the "dense" regime and the beginning of the interaction between bubbles. In this regime, the bubbles growth rate decreases in comparison with the diluted regime. The elliptical shapes develop very curved parts in the direction of the closest neighbors. Again, the higher the amount of the gelling agent, the more anisotropic the bubbles (Fig. 3).

Finally, they might enter into contact during their evolution and form thin films between them. If two bubbles in contact have a very different size, a coarsening-like behavior is observed and the small bubble decreases its size for the benefit of the largest one due to gas transfer through the thin film (Fig. 2d). Eventually, the breakage of this films result in the formation of a connected network inside the hydrogel (Fig. 2e). If the latter percolates and connects with the free interface, gas is released outside the hydrogel in a few tenths of a second. After that, the hydrogels heals and cavities close. Some smaller cavities are still present at the end where former large cavities were found (Fig. 2f)

Changing the pressure decrease rate reveals some specific behaviors of our 2D geometry. The pressure decrease rate is characterized by its average value between 250 and $150 \mathrm{mbar}$, since the pressure evolution is not linear with time. The effect of three different pressure rates are shown in Fig. 4. For the lowest pressure decrease (-0.7 mbar.s $\left.{ }^{-1}\right)$, the bubbles evolution is homogeneous whatever the position inside the cell. For the intermediate case (-1.4 mbar.s $\left.{ }^{-1}\right)$, a gradient of size appears through the cell. The closer to the free interface the bubbles, the larger their areas. This effect is even clearer and more dramatic for the higher pressure decrease rate (30 mbar. $\left.\mathrm{s}^{-1}\right)$. In that case, bubbles close to the interface percolate very quickly while bubbles in bulk remain very small. This effect may be explained by the pressure drop inside the hydrogel when the material is expanding too quickly. In what follows, imposed pressure rates will always be relatively slow, ranging between -0.33 and -0.81 mbar.s ${ }^{-1}$, such that the internal pressure could be considered as homogeneous inside the hydrogel. In that limit, we ensure that the hydrogel pressure equals the pressure at the free interface imposed by the pump and measured by our pressure sensor. 

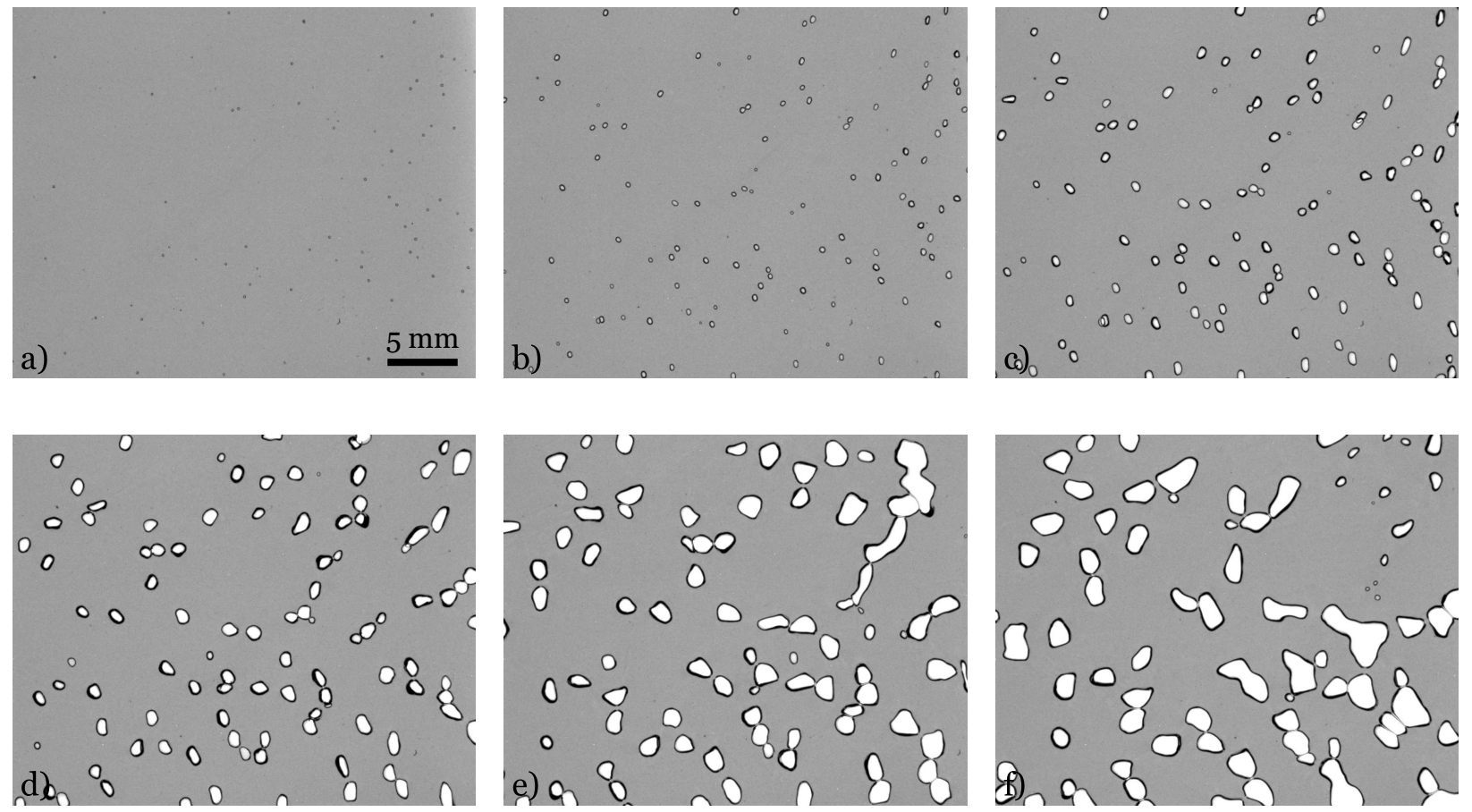

FIGURE 2. Image sequence of bubbles growth inside a $4 \%$ hydrogel with $100 \mathrm{~s}$ between two images and a pressure rate -0.67 mbar.s ${ }^{-1}$. Bubbles are advected toward the pumping direction, here on the right hand side, due to the global expansion of the hydrogel triggered by the nucleation and growth of bubbles.
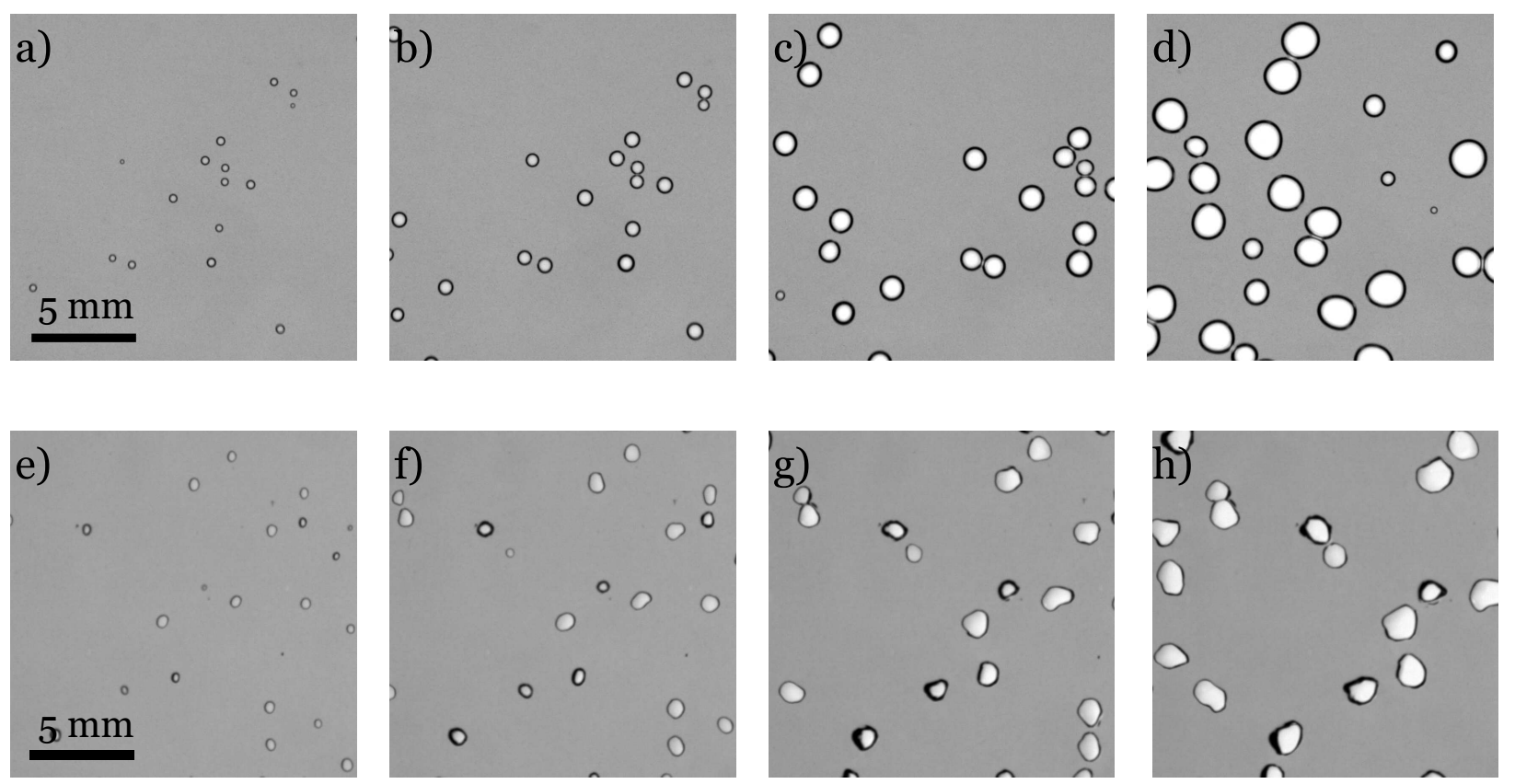

Figure 3. Image sequence of bubbles growth : (a-d) inside a 3\% hydrogel with $130 \mathrm{~s}$ between two images and a pressure rate -0.30 mbar.s ${ }^{-1}$, (e-h) inside a $5 \%$ hydrogel with $300 \mathrm{~s}$ between two images and a pressure rate $-0.45 \mathrm{mbar} / \mathrm{s}$. Pumping from the right side. 

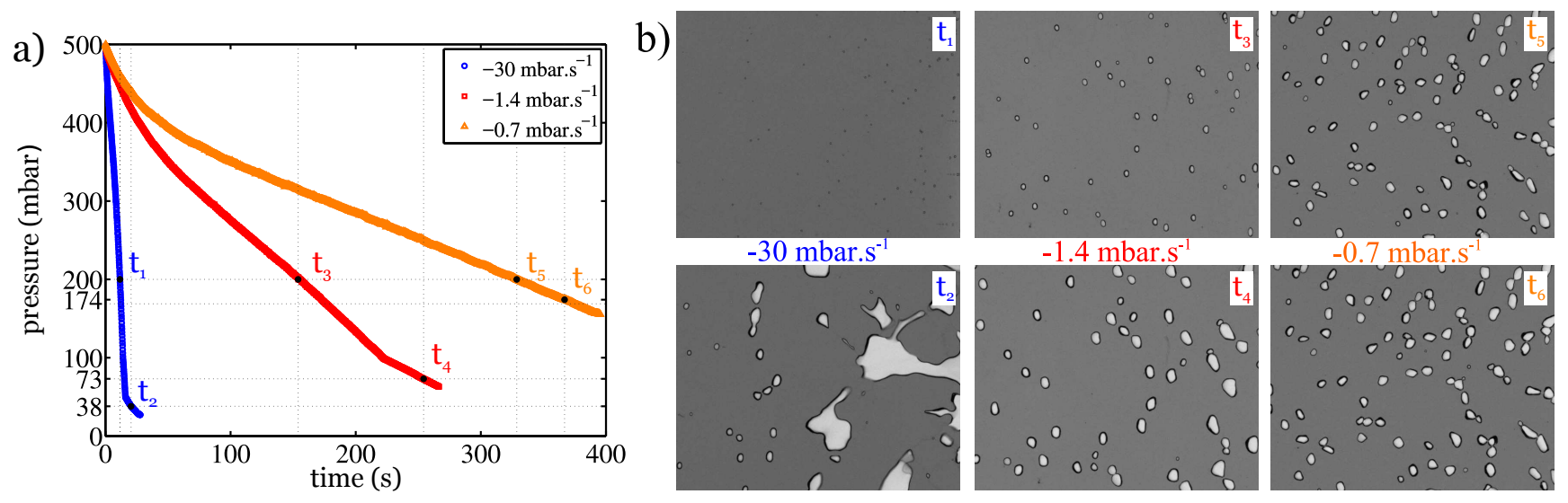

FiguRE 4. a) Imposed pressure for three different pressure decrease rates : $-30,-1.4$ and -0.7 mbar.s ${ }^{-1}$ respectively. b) Experimental snapshots obtained at the times $t_{1}, t_{2}, \ldots, t_{6}$. The hydrogel concentration is $4 \%$. The observation field is $2.6 \mathrm{~cm} \times 3.3$ cm large.

\begin{tabular}{|c|c|c|c|c|}
\hline hydrogel composition & set(s) of experiments & pressure characteristics & symbol & $\mathcal{D}\left(\mathrm{m}^{2} \cdot \mathrm{s}^{-1}\right)$ \\
\hline $3 \%$ & 1 & constant, 188 mbar & $\square$ & $(1.9 \pm 0.2) \times 10^{-10}$ \\
\hline $3 \%$ & 2 & decrease, -0.33 mbar.s ${ }^{-1}$ & $\Delta$ & $(2.88 \pm 0.02) \times 10^{-10}$ \\
\hline $3 \%$ & 2 & decrease, -0.81 mbar.s ${ }^{-1}$ & $\diamond$ & $(3.19 \pm 0.06) \times 10^{-10}$ \\
\hline $3 \%$ & $1,2,3$ & decrease, -0.45 mbar.s ${ }^{-1}$ & $\circ$ & $(3.82 \pm 0.05) \times 10^{-10}$ \\
\hline $4 \%$ & 3 & decrease, -0.45 mbar.s ${ }^{-1}$ & $*$ & $(2.13 \pm 0.04) \times 10^{-10}$ \\
\hline $5 \%$ & 3 & decrease, -0.45 mbar. $^{-1}$ & + & $(2.80 \pm 0.08) \times 10^{-10}$ \\
\hline
\end{tabular}

TABLE II. Experiments parameters and measurements : hydrogel composition, set(s) of experiments, pressure characteristics, symbols, model parameter $\mathcal{D}$ (Eq. 6).

\section{DILUTED REGIME - EXPERIMENTS}

The diluted regime is characterized by a bubble dynamics that is not influenced by the presence of the neighboring bubbles. Experiments are performed on several hydrogels and for various pressure evolutions (Tab. II). For each of them, we track the area of the bubbles. This area corresponds to the darker and outer part of the projected area of the bubble. Data are recorded as soon as the first bubble becomes measurable (area around $0.5 \mathrm{~mm}^{2}$ ) and can be tracked by an automatized procedure. For each experiment, a few tenth of bubbles inside the diluted regime are selected and tracked until they reach the crossover between the diluted and dense regimes (Sec. VI). Three sets of experiments are presented. The first one consists of a $3 \%$ hydrogel subjected to either a constant pressure or a pressure decrease. The second one consists of the same $3 \%$ hydrogel subjected to three different pressure decreases. The third one consists of three different hydrogels $(3,4$ and $5 \%)$ subjected to the same pressure decrease.

Set 1 deals with the comparison between a constant pressure experiment and a decreasing pressure experiment both performed with the same hydrogel composition 3\% (Fig. 5). For the constant pressure, the area of each bubble increases almost linearly in time. Therefore the growth rate is constant, around $0.0008 \mathrm{~mm}^{2} \cdot \mathrm{s}^{-1}$. It seems to be the same for all bubbles even with different areas, due to different nucleation times. For the decreasing pressure experiment, the growth rate seems to increase with time leading to parabola-like curves for the areas. Given that bubbles have nucleated at different times, the later the bubble is nucleated, the quicker the growth rate. Plotting the area as a function of pressure gives a significant dispersion of the data. But we observed that the data of all bubbles collapse while plotting the growth rate as a function of the pressure. This means that the growth rate is homogeneous in space for a given pressure whatever the bubble size.

Set 2 deals with the comparison between three decreasing pressure experiments with the same hydrogel composition 3\% (Fig. 6). Different pressure rate are used with a factor 2.5 between the highest and the lowest (Tab. II). For a given experiment, the data confirm that plotting the area rate $d A / d t$ as a function of the pressure $P$ allows to collapse all the experimental points on a single curve. The three curves are very similar and suggest that the bubble dynamics in the diluted regime is quasistatic in our experimental range. The small differences between them do not enable to discriminate the role or the in- 

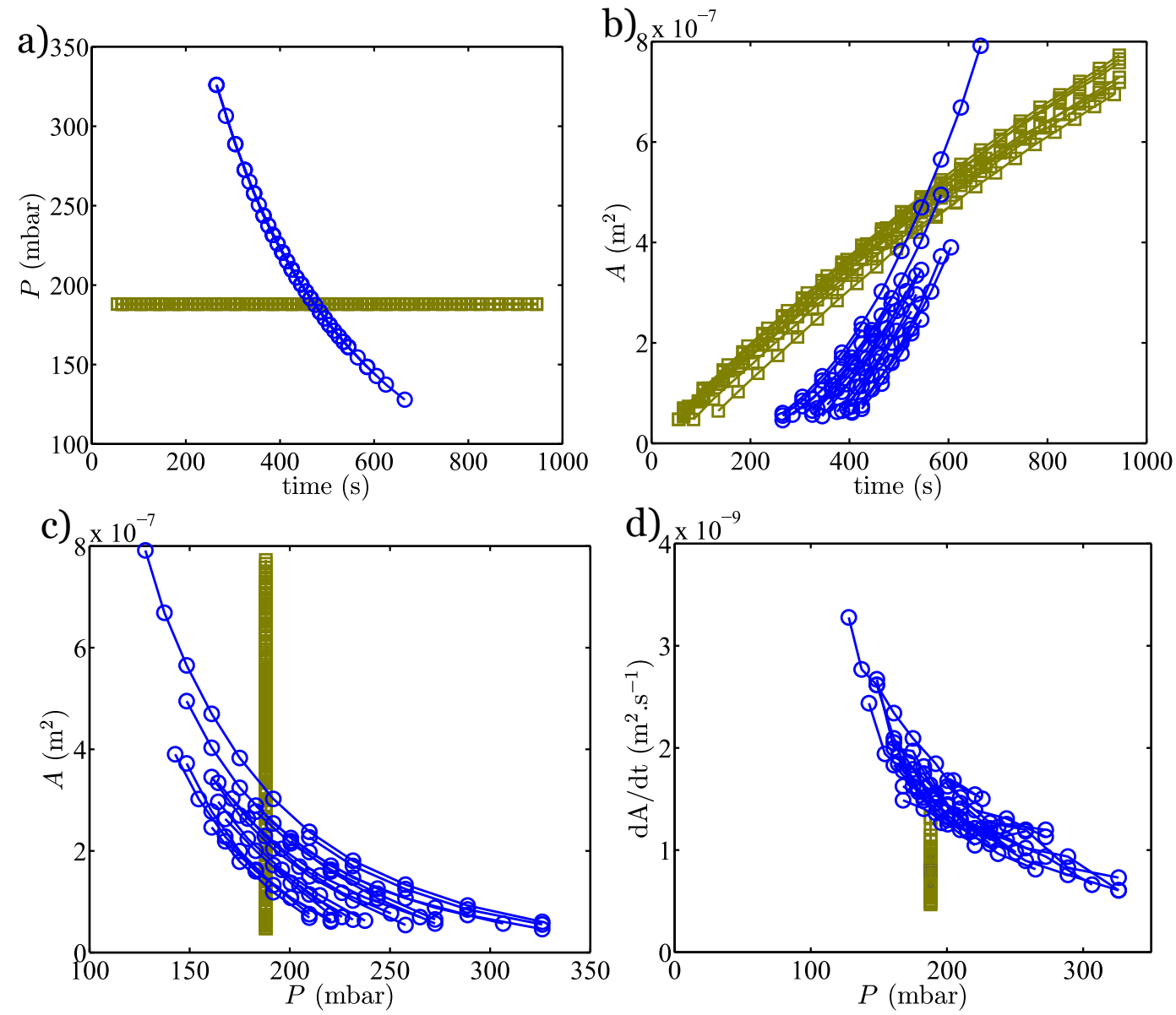

Figure 5. Diluted regime - Set 1. Constant pressure ( $\square$ ) and decreasing pressure (o) experiments with the same hydrogel composition 3\%. For both experiments, several bubbles are tracked and their respective data points are connected by solid lines. Pressure as a function of time (a), area as a function of time (b), area as a function of pressure (c) and area growth rate as a function of pressure (d). For the constant pressure experiment, all bubbles grow with a quasi constant and homogeneous growth rate. Its value is centered around $0.8 \times 10^{-9} \mathrm{~m}^{2} . \mathrm{s}^{-1}$. For the decreasing pressure experiment, the bubbles do not nucleate simultaneously and each of them follows its own area-time or area-pressure trajectory. For a given time, the growth rate is the same for all bubbles. It increases as the pressure decreases and when plotting the growth rate as a function of the imposed pressure, all the data points collapse onto the same trend.

fluence of the pressure rate (the data of the intermediate pressure rate are not found in between the ones of the two extreme pressure rates) and the effect of this parameter can not be resolved with the precision of the setup. The constant pressure experiment performed in set 1 , which can be seen as an extremely low pressure rate experiment, gives a data range that is very close to the trends obtained with the decreasing pressure experiments (not plotted in Fig. 6 for simplicity), which is consistent with the quasistatic regime hypothesis.

Set 3 deals with the comparison between three different hydrogels (compositions 3,4 and $5 \%$ ) subjected to the same pressure decrease (Fig. 6). It is worth noting that the $5 \%$ hydrogel contains initially small bubbles (Sec II). Therefore their initial areas are different from zero. Again, plotting $d A / d t$ as a function of $P$ seems to be the best choice to display a single trend for a given expe- riment. We notice that the three curves do not exactly collapse but are relatively similar while the rheological properties of the three hydrogels are rather different (up to a factor 500 for the elastic properties, Tab. I, while we have at most a factor 2 for the area rate at a given pressure). Again, the effect of this parameter can not be resolved with the precision of our setup since the data of the $4 \%$ hydrogel are not found in between the ones of the 3 and $5 \%$ hydrogels. To conclude, the composition and the rheological properties do not seem to play a significant role to account for the bubble growth rate in the diluted regime. As observed before they have a small influence on the morphology in the diluted regime, since bubbles are much more anisotropic for the highest concentrations. 

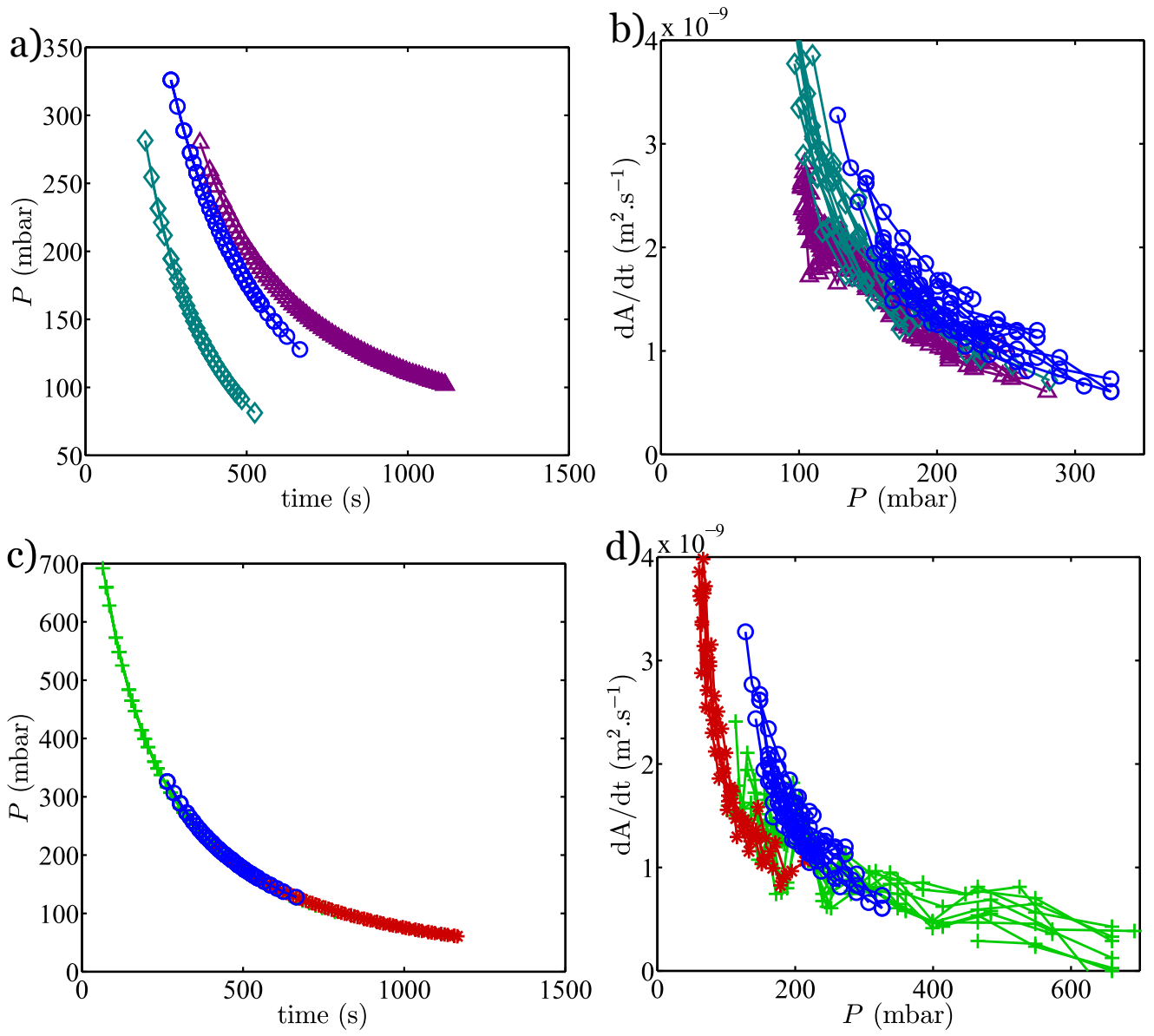

Figure 6. Diluted regime - Sets 2 and 3. (a-b) Three decreasing pressure experiments at different pressure rates $(-0.33(\triangle)$, $-0.45(\circ)$ and $-0.81(\diamond)$ mbar.s ${ }^{-1}$ ) with the same hydrogel composition $3 \%$ : pressure as a function of time (a) and area rate as a function of pressure (b). (c-d) Three hydrogel compositions (3\% (o), 4\% (*) and 5\%(+)) with the same pressure rate -0.45 mbar.s ${ }^{-1}$ : pressure as a function of time (c) and area growth rate as a function of pressure (d). For a given experiment, all data points collapse onto a single trend when plotting the growth rate as a function of the imposed pressure. By varying the pressure rate or the hydrogel composition, trends are maintained. The small differences observed do not enable to discriminate the impact of these two parameters on the evolution of the growth rate (see text).

\section{DILUTED REGIME - MODEL}

The experiments described previously suggest that the bubble growth rate is a function only of the pressure and does not depend neither on the bubble area nor on the pressure rate nor on the rheological properties of the hydrogel : the smaller the pressure, the larger the growth rate. We propose a model to account for these observations. It assumes that the bubble growth is mainly driven by the diffusion of the dissolved gas through the hydrogel and its outgassing at the reactive-advected hydrogel-gas interface.

We first assume that the pressure is uniform through the whole hydrogel and inside the gas bubbles. As observed previously, the pressure loss through the hydrogel is small when the pressure rate is small enough. By taking a surface tension of order $10^{-2} \mathrm{~N} . \mathrm{m}^{-1}$ and a bubble radius around $0.1-1 \mathrm{~mm}$, the Laplace pressure at the hydrogel-bubble interface is found around 10-100 Pa and is negligible compared to the absolute pressure inside the hydrogel. Following these considerations the pressure $P(t)$, which is the control parameter, is uniform and is the same inside the hydrogel and inside the bubbles. The temperature change is around $3 \%$ so that we will assume a constant temperature in the model.

Given the Hele-Shaw geometry, we expect a 2D-3D geometry crossover when the bubble radius reaches 0.5 $\mathrm{mm}$ or when the projected area reaches $A_{c}=0.8 \mathrm{~mm}^{2}$ equivalently. In most of the data, $A<A_{c}$ and the $3 \mathrm{D}$ problem has to be considered. Nevertheless, bubbles might nucleate close to one of the glass plates and trigger specific confining effects. As seen below, the 3D approach seems to capture the main features of the growth dynamics, even though some corrections could be introduced by considering the confinement, which is out of the scope of our study. In the diluted regime, all bubbles be- 
have independently and we consider only the case of a single bubble with the spherical geometry. The bubble is centered at $r=0$ in the spherical coordinates reference frame. We note $R$ its radius, $V=4 \pi R^{3} / 3$ its volume and $A=\pi R^{2}$ its projected area.

The evolution of the dissolved gas concentration $c(r, t)$ (units of mol. $\mathrm{m}^{-3}$ ) at a distance $r>R$ in the hydrogel is given by the advection-diffusion equation :

$$
\frac{\partial c}{\partial t}+\vec{u} \cdot \vec{\nabla} c=D \Delta c
$$

where $D$ is the diffusion coefficient of the dissolved gas in the hydrogel and $\vec{u}$ is the velocity produced in the hydrogel by bubble growth. The initial gas concentration is noted $c_{0}$. It corresponds to the saturation concentration in equilibrium with the initial pressure $P_{0}$ (here the room pressure for our experiments, approximated by 1000 mbar). Boundary conditions are $c(r, 0)=c_{0}$ for $r>R(0)$ and $c(r, t)=c_{0}$ for $r \rightarrow \infty$ and $t>0$. Due to the outgassing at the hydrogel-bubble interface, the concentration at $r=R$ is the saturation concentration in equilibrium with the pressure inside the bubble (note that it corresponds to the imposed pressure $P$ that is uniform through the whole hydrogel). We note $c(R(t), t)=c_{s}(t)$ for $t>0$. Solving the advection-diffusion equation is not an easy task. Following Epstein and Plesset [15], we can get a reasonable physical approximation by neglecting first the advection term, solving the diffusion equation and make a coordinate change in a second step to account for the moving boundary condition. In that case, the concentration gradient at the moving boundary is written :

$$
\left(\frac{\partial c}{\partial r}\right)_{r=R}=\frac{c_{0}-c_{s}}{R}\left(1+\frac{R}{\sqrt{\pi D t}}\right)
$$

As suggested in the same article, we have checked that $R / \sqrt{\pi D t}<<1$ (this factor equals 0.25 at most in our experiments - data not shown). This supports the physical approximation made above and shows that $R$ is the relevant length scale of the spatial concentration variations around a bubble.

\begin{tabular}{|c||c|c|c|}
\hline Gas & D & $H$ & $\mathcal{D}$ \\
\hline & $\mathrm{m}^{2} \mathrm{~s}^{-1}$ & $\mathrm{~mol}^{-\mathrm{Pa}^{-1}} \cdot \mathrm{m}^{-3}$ & $\mathrm{~m}^{2} \cdot \mathrm{s}^{-1}$ \\
\hline \hline $\mathrm{N}_{2}$ & $1.9 \times 10^{-9}$ & $6.1 \times 10^{-6}$ & $4.6 \times 10^{-12}$ \\
\hline $\mathrm{O}_{2}$ & $2.1 \times 10^{-9}$ & $1.3 \times 10^{-5}$ & $1.1 \times 10^{-11}$ \\
\hline $\mathrm{Ar}$ & $2.0 \times 10^{-9}$ & $1.4 \times 10^{-5}$ & $1.1 \times 10^{-11}$ \\
\hline $\mathrm{CO}_{2}$ & $1.9 \times 10^{-9}$ & $3.4 \times 10^{-4}$ & $2.6 \times 10^{-10}$ \\
\hline
\end{tabular}

TABle III. Diffusion coefficient $D$, Henry's constant $H$ and the constant $\mathcal{D}$ found in the model for nitrogen, oxygen, argon and carbon dioxide gases in water solution at $25^{\circ} \mathrm{C}$ $[22,23]$. For the calculations of $\mathcal{D}, \mathcal{R} T$ is taken equal to 2500 $\mathrm{Pa} \cdot \mathrm{m}^{3} \cdot \mathrm{mol}^{-1}$.

The amount of substance rate entering into the bubble

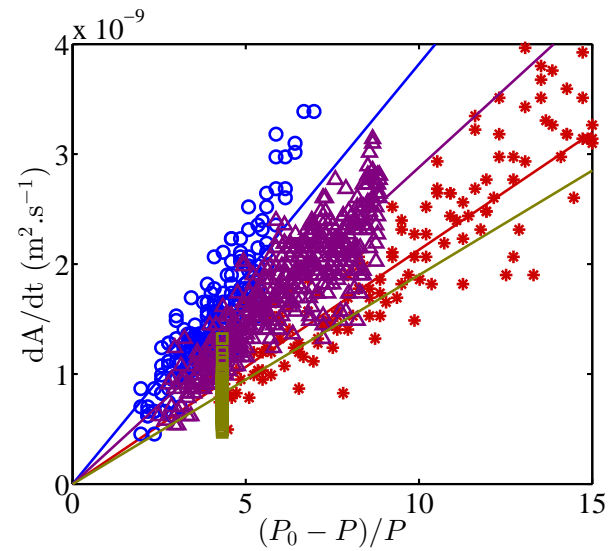

Figure 7 . Area rate $d A / d t$ vs $\left(P_{0}-P\right) / P$. For simplicity, only four sets of data are plotted (Tab. II). All time-steps for every bubble of each experiment are represented. Measurements are interpolated by straight lines modeled by Eq. 6 with $P_{0}=$ 1000 mbar and the best fits for the parameter $\mathcal{D}$.

is given by

$$
\frac{d n}{d t}=4 \pi R^{2} D\left(\frac{\partial c}{\partial r}\right)_{r=R} \simeq 4 \pi R^{2} D \frac{c_{0}-c_{s}}{R}
$$

where $n$ is the amount of substance (units of mol) inside the bubble.

At the same time, the amount of substance balance equation leads to

$$
\frac{d n}{d t}=4 \pi R^{2} \frac{n}{V} \frac{d R}{d t}=4 \pi R^{2} \frac{P}{\mathcal{R} T} \frac{d R}{d t}
$$

where we take the ideal gas equation $P V=n \mathcal{R} T$ inside the bubble and assume $d n / d V \simeq n / V$ or equivalently that the pressure is a slowly decreasing function of time and the temperature is constant. From the former equations we deduce

$$
R \frac{d R}{d t}=D \mathcal{R} T \frac{c_{0}-c_{s}}{P}=D \mathcal{R} T H \frac{P_{0}-P}{P}
$$

where $c_{0}=H P_{0}$ and $c(R(t), t)=H P(t)$ are given by the Henry's law and its solubility constant $H$. In terms of the projected area $A=\pi R^{2}$, we write

$$
\frac{d A}{d t}=\mathcal{D}\left(\frac{P_{0}-P}{P}\right)
$$

with $\mathcal{D}=\frac{D \mathcal{R} T H}{2 \pi}$ the relevant parameter. Note that $\mathcal{D}$ has the dimension of a diffusion coefficient as well. This relation is tested for every experiment with $P_{0}=1000$ mbar. For a given experiment, all data points are in agreement with Eq. 6 and an experimental measurement of the proportionality factor $\mathcal{D}$ is obtained by a linear fit (Fig. 7). Results are summarized in Tab. II. All values are comparable and range between 1.9 and $3.8 \times 10^{-10} \mathrm{~m}^{2} \cdot \mathrm{s}^{-1}$. The 


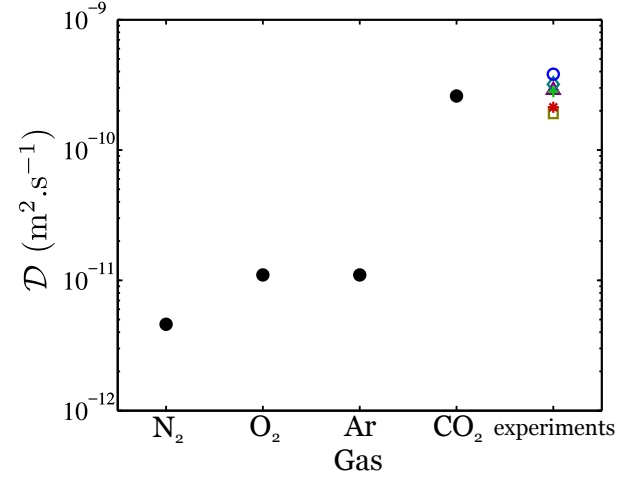

Figure 8. Model parameter $\mathcal{D}$ for gases in the air composition (black disks and Tab. III) and measured in the experiments (see Tab. II for symbols).

values of the diffusion coefficients and Henry's constants of air gases dissolved in a water solution at room pressure and temperature are summarized in Tab. III. We observe that carbon dioxide has the largest value for $\mathcal{D}$ and should correspond to the fastest outgassing gas in a water solution. Its theoretical value $2.6 \times 10^{-10} \mathrm{~m}^{2} \cdot \mathrm{s}^{-1}$ is in very good agreement with the values found experimentally with our hydrogels (Fig. 8) and shows that carbon dioxide is the gas at play in our experiments as well. Small differences in the value of $\mathcal{D}$ are observed for the same hydrogel and the three decreasing pressure rates. As expected the pressure rate is not a significant parameter. It is difficult to conclude if the slight differences are linked to the dispersion of the data or to a slight sensitivity to the experimental protocol. It might be that the hydrogel composition plays a little role on the coefficient $\mathcal{D}$ as well since 4 and $5 \%$ hydrogels have smaller values than $3 \%$ hydrogels (Tab II). Possibly this could come from the deviation from sphericity assumed in the model or slight changes in the value of the diffusion coefficient $D$ due to a change in the chemical composition. At this stage, with the dispersion of the data, it is not possible to draw strong conclusions about this effect.

Modeling the deviation from sphericity observed in 4 and $5 \%$ hydrogels inside the diluted regime would require to account for the hydrogel rheology and to calculate the stress, strain and strain rate tensors around the bubbles. The larger bubble growth in the direction perpendicular to the pulling direction is redolent of the behavior of a soft inclusion inside an elastic material under uniaxial tension [24] : the whole visco-elasto-plastic behavior of the material should be accounted for to accurately model this effect.

\section{DENSE REGIME}

When bubbles are too close, they begin to interact and enter into what we define the dense regime. The interaction is two-fold and has characteristics both on the bubble growth dynamics and morphologies. First, bubbles compete for the same dissolved gas and the concentration field calculated previously for the case of isolated bubbles does not hold anymore. As a consequence, the growth rate decreases in comparison to what is expected in the diluted regime (data not shown). Second, the stress fields in the hydrogel around bubbles overlap, resulting in a deviation from the elliptical shape observed in the diluted regime. We expect that a change in the bubble morphology might also alter the bubble growth rate as it would modify the dissolved gas concentration field.

The effect of the interaction on the bubble morphology is characterized by the deformation and the growth of very curved parts toward the position of the neighboring bubble (Fig. 9). To perform quantitative measurements, we have considered pairs of bubbles only with the following criteria : 1) they are far enough from other bubbles so that we can assume a two bodies interaction and neglect the effect of surrounding bubbles - 2) their areas are relatively similar at least inside the diluted regime. For each pair of bubbles and for each time step, we have measured the radii from the bubble centers, $r_{1}(\theta)$ and $r_{2}(\theta)$ respectively, as a function of the angular position $\theta$. For each of them, 360 values were taken. $\left\langle r_{1}\right\rangle$ and $\left\langle r_{2}\right\rangle$ denote the angular average values. For each time step, the morphology correlation function $\mathcal{C}(\alpha, t)$ is defined as the dimensionless function :

$\mathcal{C}(\alpha, t)=\frac{1}{360} \sum_{\theta=1^{\circ}}^{\theta=360^{\circ}} \frac{\left(r_{1}(\theta)-<r_{1}>\right)}{<r_{1}>} \times \frac{\left(r_{2}(\theta+\alpha)-<r_{2}>\right)}{<r_{2}>}$.

A typical angle-time diagram is given in Fig. 10a. It exhibits a band-like feature with maximal and minimal values. For times shorter than $185 \mathrm{~s}$, the maxima are centered around $0 \mathrm{deg}$ and $180 \mathrm{deg}$, and the minima are found in between. Both maxima and minima are more and more exacerbated as time proceeds and bubbles grow. This marks an increase of the bubble anisotropy. The values of the maxima, found at $\alpha=0 \mathrm{deg}$ and $180 \mathrm{deg}$, are similar (Fig. 10b). These features are characteristics of the diluted regime and the ellipse-like geometry of the bubbles. Above $185 \mathrm{~s}$, the features change significantly and the difference between maxima and minima decreases. The trends of $\mathcal{C}$ for both $0 \mathrm{deg}$ and $180 \mathrm{deg}$ are not similar anymore : the bubbles correlate now at most when oriented in opposite directions, pointing toward each other. This marks the onset of the dense regime. The bands in the angle-time diagram are not horizontal anymore and reflect the evolution of interacting bubbles into more complex geometries.

In Fig. 11, the center-to-center distance $\ell_{c c}$ between the two bubbles is plotted as a function of their radius $R$ at the onset of the dense regime. Based on the morphological criterion, we have studied the effect of the composition. For a given hydrogel, the higher the area of the bubbles, the larger the distance $\ell_{c c}$ for the crossover between the 

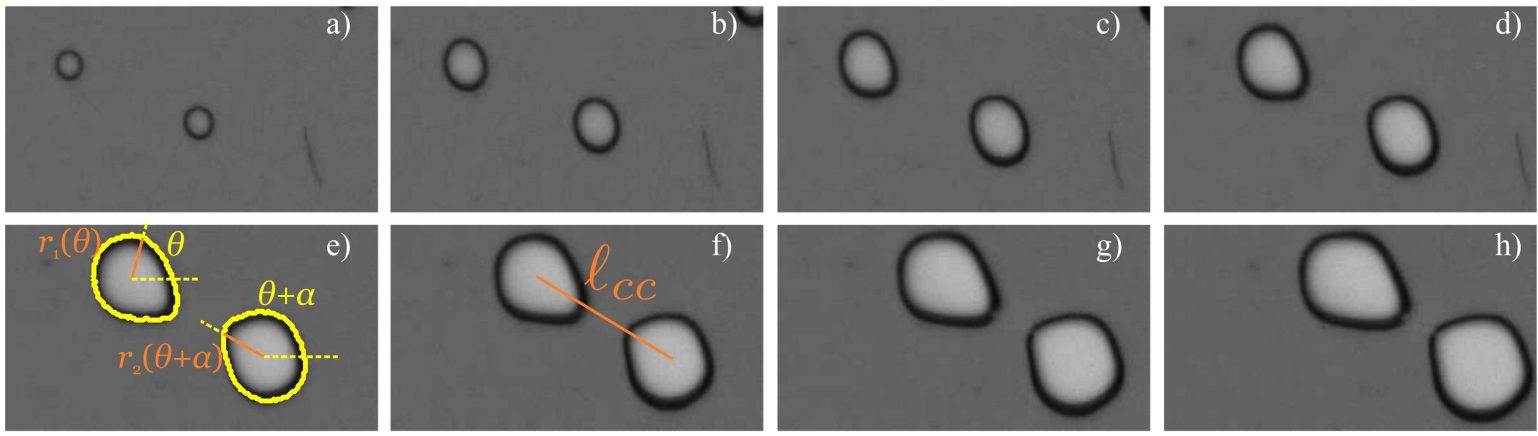

FiguRE 9. Experimental snapshots of two close-neighbor growing bubbles : a) $t=50 \mathrm{~s}, \mathrm{~b}) \mathrm{t}=100 \mathrm{~s}, \mathrm{c}) \mathrm{t}=150 \mathrm{~s}, \mathrm{~d}) \mathrm{t}=200 \mathrm{~s}, \mathrm{e}$ ) $\mathrm{t}=250 \mathrm{~s}, \mathrm{f}) \mathrm{t}=300 \mathrm{~s}, \mathrm{~g}) \mathrm{t}=350 \mathrm{~s}$ and $\mathrm{h}) \mathrm{t}=400 \mathrm{~s}$. Outer contours, bubble radii for given angles and the center-to-center distance $\ell_{c c}$ are indicated. Very curved parts begin to grow on the contour of the bubbles between $\mathrm{t}=150$ and $200 \mathrm{~s}$; this marks the onset of the bubble interaction. The hydrogel concentration is $4 \%$ and the pressure is constant, 100 mbar. The observation field is $2.5 \mathrm{~mm} \times 4.6 \mathrm{~mm}$ large.
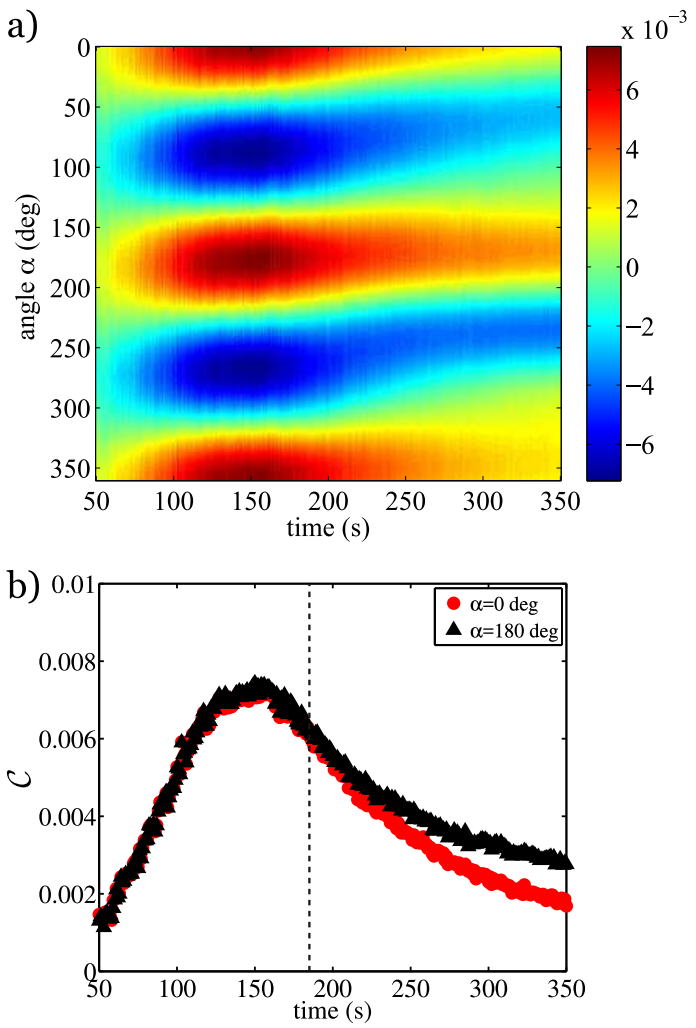

FiguRE 10. Morphology correlation measurements for the two bubbles of Fig. 9. a) Angle-time diagram of the correlation function $\mathcal{C}(\alpha, t)$. b) $\mathcal{C}$ versus time for two specific values of the angle $\alpha: 0 \mathrm{deg}$ (red circles) and $180 \mathrm{deg}$ (black triangles) respectively. The crossover is observed around $185 \mathrm{~s}$ (vertical dashed line).

diluted and dense regimes. The correlation does not seem to depend significantly on the hydrogel composition and rheological properties. The data are rather dispersed but a linear interpolation gives $\ell_{c c} \simeq 4.3 R$. It means that the

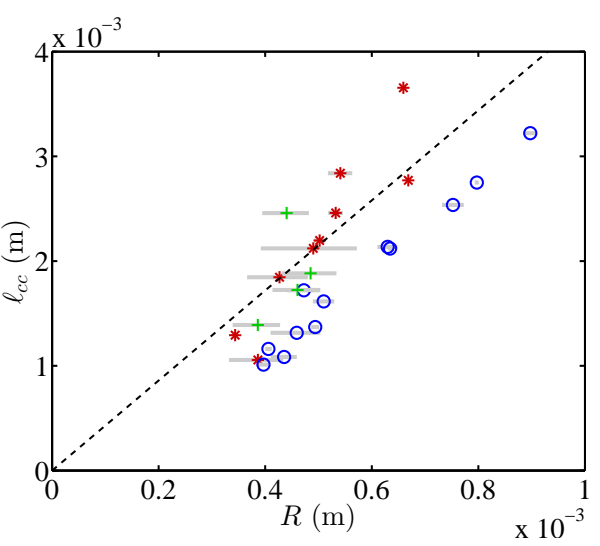

Figure 11. Center-to-center distance $\ell_{c c}$ as a function of the bubble radius $R$ for pairs of bubbles at the crossover between the diluted and dense regimes. The criterion is based either on the bubble morphology for hydrogels of composition $3 \%$ (०), $4 \%(*)$ and $5 \%(+)$. Error bars are plotted in light grey and are inferred from the area difference between the two interacting bubbles. The dashed line represents $\ell_{c c}=4.3 R$.

bubbles begin to interact at a distance comparable to the bubble size regardless of the hydrogel composition. A model of the bubble interaction is still missing to account for the observations.

\section{CONCLUSION}

The decreasing pressure experiment proposed in this study has provided insights about the bubble dynamics inside an outgassing hydrogel. Carbon dioxide is the gas at play and such a study should help to understand some aspects of carbon dioxide sequestration in non-Newtonian fluids. We have focused on both the growth rate and the morphology of the bubbles and have 
identified a crossover between a diluted regime of isolated bubbles and a dense regime where bubbles interact. Other rheological behaviors should be studied in the future and performing the study to smaller length scales should exhibit surface tension effects. Models should also be derived to account for the gas concentration and stress

[1] E. Bouchbinder, T.-S. Lo, and I. Procaccia, "Dynamic failure in amorphous solids via a cavitation instability," Phys. Rev. E, vol. 77, p. 025101, Feb. 2008.

[2] H. Lakrout, P. Sergot, and C. Creton, "Direct Observation of Cavitation and Fibrillation in a Probe Tack Experiment on Model Acrylic Pressure-Sensitive-Adhesives," The Journal of Adhesion, vol. 69, pp. 307-359, Mar. 1999.

[3] I. Chikina and C. Gay, "Cavitation in Adhesives," Phys. Rev. Lett., vol. 85, pp. 4546-4549, Nov. 2000.

[4] G. W. Scherer and D. M. Smith, "Cavitation during drying of a gel," Journal of Non-Crystalline Solids, vol. 189, pp. 197-211, Sept. 1995.

[5] J. A. Zimberlin, N. Sanabria-DeLong, G. N. Tew, and A. J. Crosby, "Cavitation rheology for soft materials," Soft Matter, vol. 3, pp. 763-767, May 2007.

[6] S. Kundu and A. J. Crosby, "Cavitation and fracture behavior of polyacrylamide hydrogels," Soft Matter, vol. 5, no. 20, p. $3963,2009$.

[7] J. A. Zimberlin, J. J. McManus, and A. J. Crosby, "Cavitation rheology of the vitreous : mechanical properties of biological tissue," Soft Matter, vol. 6, pp. 3632-3635, July 2010.

[8] D. Palanker, I. Turovets, and A. Lewis, "Dynamics of ArF excimer laser-induced cavitation bubbles in gel surrounded by a liquid medium," Lasers Surg. Med., vol. 21, pp. 294-300, Jan. 1997.

[9] P. Babin, G. Della Valle, H. Chiron, P. Cloetens, J. Hoszowska, P. Pernot, A. L. Réguerre, L. Salvo, and R. Dendievel, "Fast X-ray tomography analysis of bubble growth and foam setting during breadmaking," Journal of Cereal Science, vol. 43, pp. 393-397, May 2006.

[10] A. Turbin-Orger, P. Babin, E. Boller, L. Chaunier, H. Chiron, G. D. Valle, R. Dendievel, A. L. Réguerre, and L. Salvo, "Growth and setting of gas bubbles in a viscoelastic matrix imaged by $\mathrm{X}$-ray microtomography : the evolution of cellular structures in fermenting wheat flour dough," Soft Matter, vol. 11, pp. 3373-3384, Apr. 2015.

[11] C. A. Villamizar and C. D. Han, "Studies on structural foam processing II. Bubble dynamics in foam injection molding," Polym Eng Sci, vol. 18, pp. 699-710, July 1978.

[12] T. Divoux, E. Bertin, V. Vidal, and J.-C. Géminard, "Intermittent outgassing through a non-Newtonian fluid," fields overlap in the dense regime.

\section{ACKNOWLEDGMENTS}

This project was supported by the "Agence Nationale de la Recherche" (CAVISOFT : ANR-2010-JCJC-0407 01).
Phys. Rev. E, vol. 79, p. 056204, May 2009.

[13] N. G. Lensky, R. W. Niebo, J. R. Holloway, V. Lyakhovsky, and O. Navon, "Bubble nucleation as a trigger for xenolith entrapment in mantle melts," Earth and Planetary Science Letters, vol. 245, pp. 278-288, May 2006.

[14] G. Liger-Belair, G. Polidori, and P. Jeandet, "Recent advances in the science of champagne bubbles," Chem. Soc. Rev., vol. 37, pp. 2490-2511, Nov. 2008.

[15] P. S. Epstein and M. S. Plesset, "On the Stability of Gas Bubbles in Liquid-Gas Solutions," The Journal of Chemical Physics, vol. 18, no. 11, p. $1505,1950$.

[16] E. Barlow and W. Langlois, "Diffusion of Gas from a Liquid into an Expanding Bubble," IBM Journal of Research and Development, vol. 6, pp. 329-337, July 1962.

[17] J. R. Street, A. L. Fricke, and L. P. Reiss, "Dynamics of Phase Growth in Viscous, Non-Newtonian Liquids. Initial Stages of Growth," Ind. Eng. Chem. Fund., vol. 10, pp. 54-64, Feb. 1971.

[18] R. D. Patel, "Bubble growth in a viscous Newtonian liquid," Chemical Engineering Science, vol. 35, no. 11, pp. 2352-2356, 1980.

[19] C. D. Han and H. J. Yoo, "Studies on structural foam processing. IV. Bubble growth during mold filling," Polym Eng Sci, vol. 21, pp. 518-533, June 1981.

[20] A. Arefmanesh, S. G. Advani, and E. E. Michaelides, "A numerical study of bubble growth during low pressure structural foam molding process," Polym Eng Sci, vol. 30, pp. 1330-1337, Oct. 1990.

[21] H. Tabuteau, S. Mora, G. Porte, M. Abkarian, and C. Ligoure, "Microscopic Mechanisms of the Brittleness of Viscoelastic Fluids," Phys. Rev. Lett., vol. 102, p. 155501, Apr. 2009.

[22] E. L. Cussler, Diffusion : Mass Transfer in Fluid Systems. Cambridge University Press, Jan. 2009.

[23] R. Sander, "Compilation of Henry's law constants (version 4.0) for water as solvent," Atmospheric Chemistry and Physics, vol. 15, pp. 4399-4981, Apr. 2015.

[24] S. Timoshenko and J. N. Goodier, Theory of elasticity. New York : McGraw-Hill, 1951. 